\title{
Validation and refinement of a RUNX1 mutation-associated gene expression signature in blast crisis chronic myeloid leukemia
}

(c) The Author(s) 2022

Leukemia (2022) 36:892-896; https://doi.org/10.1038/s41375-02201508-1

\section{TO THE EDITOR:}

We note with great interest recent work by Awad et al. [1]. that has identified specific $R U N X 1$ mutation-related gene expression signatures in blast crisis $(\mathrm{BC})$ chronic myeloid leukemia (CML) patient samples harbouring RUNX1 mutations. Recurrent mutations in the RUNX1 tumour suppressor gene are one of the most frequent events in $B C$ $\mathrm{CML}$ and are associated with disease progression, poor response and adverse patient outcomes [2]. Mechanistically, RUNX1 mutations that decrease transcriptional activity or exert a dominant negative effect on wild type RUNX1 are thought to block differentiation leading to BC or accelerated phase-like phenotypes in mice [3].

Consequently, new treatment options such as with CD19-CAR $T$ cells, mTOR, BCL2 and VEGFR targeted therapies identified by the authors become important avenues for RUNX1 mutant BC CML patients. Therefore, it is imperative to accurately diagnose and assess the suitability of $B C$ CML patients for new therapeutic approaches and identify additional molecular vulnerabilities that can improve patient outcomes.

In light of this, we examined our own cohort of BC CML patients [4] to identify cases with RUNX1 mutations and assess some of their molecular features compared to wild type RUNX1 cases. We recently performed a whole genome and transcriptome-wide study of BC CML patients in bulk bone marrow mononuclear cells and CD34+ leukemic stem cell populations. We identified RUNX1 aberrations in one lymphoid blast crisis (LBC) and three myeloid blast crisis (MBC) patients. Of these, two MBC cases and oneLBC harbour point mutations in the Runt homology domain that affect DNA binding (Fig. 1A). The remaining MBC patient possesses the $t(3 ; 21)$ translocation resulting in the RUNX1-MECOM fusion known to exert a dominant negative effect on normal RUNX1 transcriptional activity [5].

Here, we first confirm that the upregulated genes of the RUNX1 mutation transcriptional signature ('Awad' signature) enrich significantly (Fig. 1B, Supplementary Tables 1 and 2) in our RUNX1 mutant $B C$ samples in gene set enrichment analysis (GSEA [6]). For downregulated genes of the Awad signature, there were no significant enrichments (Supplementary Tables 1 and 2), and we did not make further use of these genes in this regard as their expression were low or undetectable in our microarrays. Similar results were obtained when we restricted the GSEA analysis to only protein coding genes within the Awad signature (Supplementary Table 3).
Notably, we also found that the upregulated Awad signature was enriched in wild type RUNX1 CD34 + LBC cases. Three wild type RUNX1 CD34 + LBC samples showed consistent enrichment for upregulated genes while a fourth sample that was biphenotypic did not show any enrichment (Fig. 1B). To confirm that the Awad signature does indeed significantly co-detect wild type RUNX1 CD34 + LBC samples and not just RUNX1 mutant samples, we compared $\mathrm{CD} 34+\mathrm{LBC}$ against $\mathrm{CD} 34+\mathrm{MBC}$ where none of the samples carry any RUNX1 mutations (Fig. $1 C$ and Supplementary Table 2). Indeed, wild type RUNX1 CD34 + LBC samples were enriched for the upregulated Awad signature genes compared to wild type RUNX1 CD34 + MBC samples.

Subsequently, we sought to develop more discriminating gene expression signatures and reasoned that direct targets of RUNX1 and genes sets from other studies may be able to detect differences between RUNX1 mutant versus wild type patients more specifically. We screened and identified 24 gene sets from the CISTROME database [7] curated from RUNX1 ChIP-seq experiments with a focus on human leukemic and hematopoietic studies and 42 gene sets from MSigDB v7.3 [6] for RUNX1regulated gene expression with RUNX2 and RUNX3 gene sets as negative controls (Supplementary Table 1). We found that some gene sets were able to identify RUNX1-mutated BC samples (Fig. 1D and Supplementary Table 2) without significantly detecting wild type RUNX1 CD34 + LBC samples (Fig. 1E and Supplementary Table 2). To confirm this, we used these gene sets to make a control comparison between wild type RUNX1 CD34+ LBC and the MBC equivalent, and found no significant concordant enrichments, or there were depletions instead (Fig. 1D, F and Supplementary Table 2).

While the Awad signature co-detects lymphoid genes in wild type RUNX1 LBC, it may still be able to detect RUNX1 mutationrelated signatures that are distinct from LBC identity per se. Indeed, GSEA analysis of 1 mutant RUNX1 CD34 + LBC against 3 wild type RUNX1 CD34 + LBCs showed the Awad and shortlisted $R U N X 1$ gene sets were significantly enriched (FDR $<0.25)$ in the mutant RUNX1 CD34 + LBC sample (except for MORF_RUNX1 which enriched in the correct direction but did not achieve significance [Fig. 2A and Supplementary Table 4]). We conclude that RUNX1 mutation gene sets can be used to detect RUNX1 mutation-containing samples in both $\mathrm{MBC}$ and $\mathrm{LBC}$, but only when comparing within separate $M B C$ and $L B C$ datasets.

As a further test of the utility of $R U N X 1$ mutational signatures to detect RUNX1 targets within leukemic transcriptomes, we next examined a large cohort of 151 AML patients with RNA-Seq data from TCGA [8] (Supplementary Table 5). When tested against mutant and wild type RUNX1 TCGA AML patients, both the Awad 
A

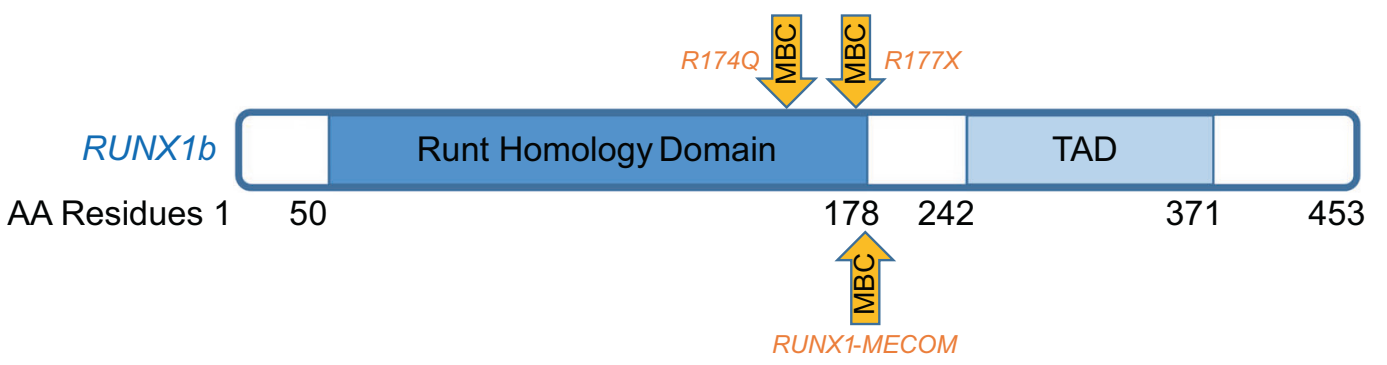

B

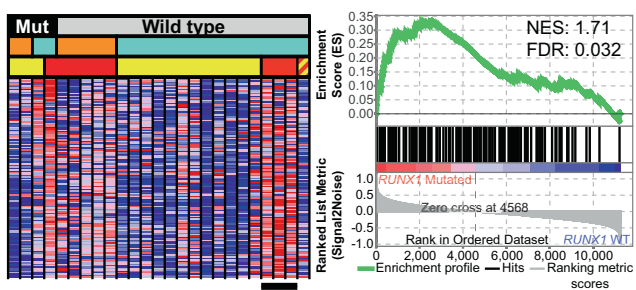

C

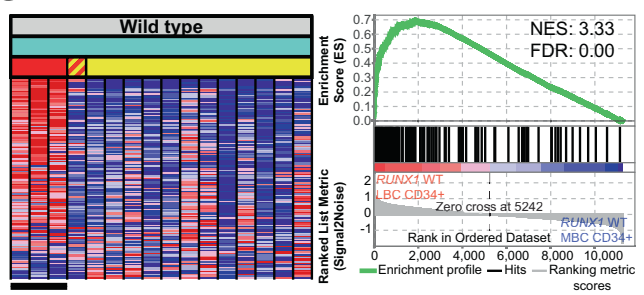

248 Gene Upregulated RUNX1 Mutation Signature

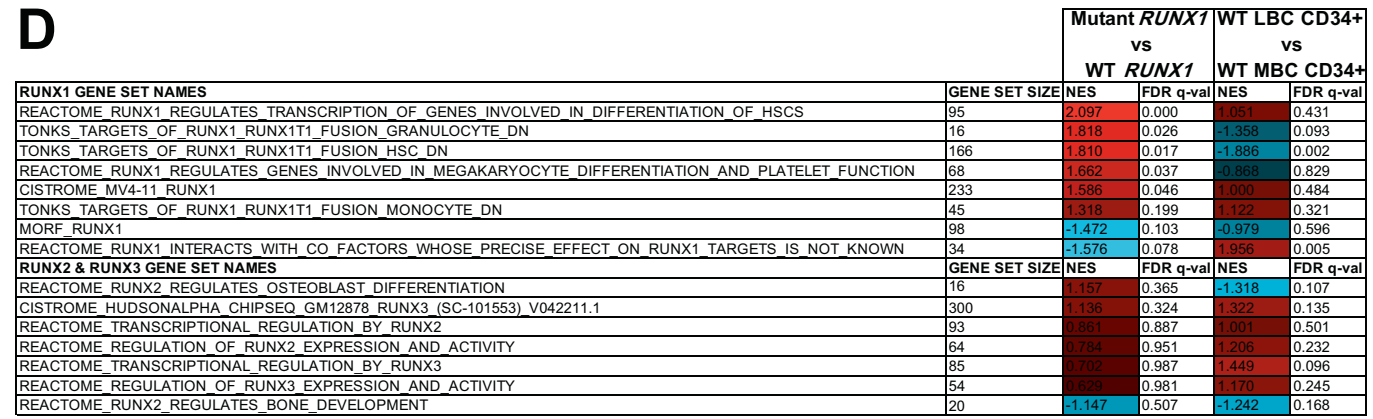

E
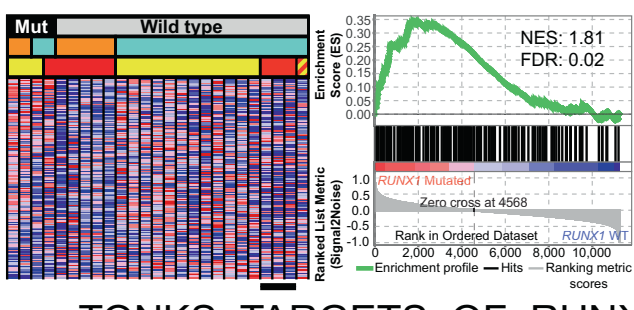

$\mathbf{F}$

TONKS_TARGETS_OF_RUNX1_RUNX1T1_FUSION_HSC_DN

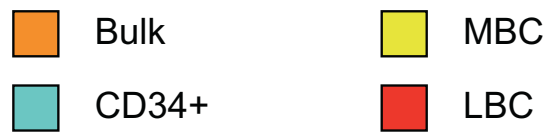

Biphenotypic MBC/LBC

Fig. 1 Validation and refinement of RUNX1 mutational signatures in CML blast crisis patients. A Schema of $R U N X 1 b$ gene isoform with mutations found in 3 MBC and 1 LBC CML patients. Horizontal scale indicates amino acid (aa) residue positions. B GSEA heatmap in left panel shows expression of genes upregulated in the Awad RUNX1 mutational signature in RUNX1 mutant (Mut) compared to wild type patients in red for upregulation and blue for downregulation. Right panel shows enrichment plot with normalised enrichment score (NES) and false discovery rate (FDR). C GSEA heatmap in left panel and enrichment plot in right panel shows enrichment of the same gene set in wild type RUNX1 CD34 + LBC patient gene expression data compared to the equivalent in MBC. D Table shows alternative gene sets that can significantly distinguish mutant RUNX1 blast crisis samples from wild type (WT) in the top half (FDR $<0.25$ ). The same gene sets do not enrich significantly for wild type RUNX1 CD34 + LBC samples compared to the equivalent in MBC or are depleted. Negative control RUNX2 and RUNX3 gene sets also do not enrich significantly in RUNX1 mutant compared to wild type samples in the bottom half. E GSEA heatmap in left panel shows expression of the TONKS_TARGETS_OF_RUNX1_RUNX1T1_FUSION_HSC_DN gene set [11] with enrichment in RUNX1 mutant (Mut) compared to wild type patients. Right panel shows enrichment plot with normalised enrichment score (NES) and false discovery rate (FDR). F GSEA heatmap in left panel and enrichment plot in right panel shows depletion of the same gene set in wild type RUNX1 CD34 + LBC compared to the equivalent MBC gene expression data. Black bar indicates 3 CD34+ wild type RUNX1 LBC samples while yellow and red hatched bar denotes a biphenotypic wild type RUNX1 sample with characteristics of both LBC and MBC. 

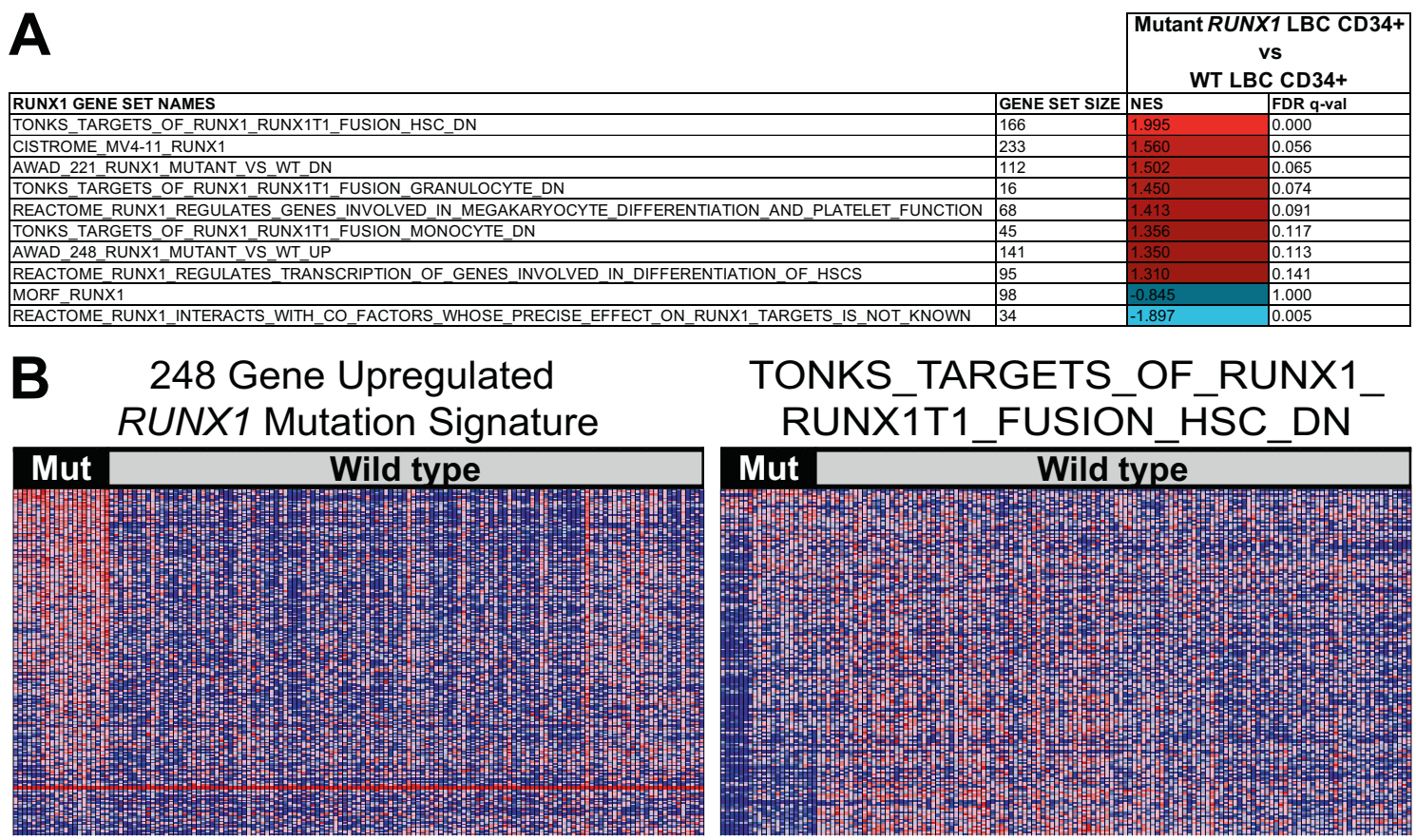

C 248 Gene Upregulated RUNX1 Mutation Signature

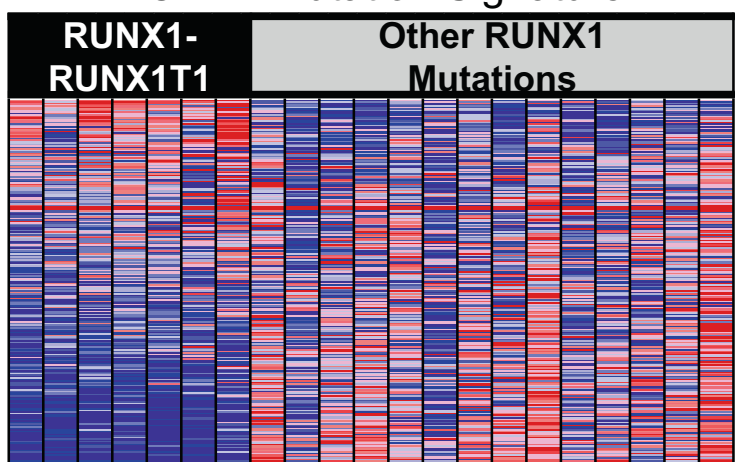

D

Pathway Analysis of Mutant RUNX1 Lymphoid Genes Expressed Predominantly in MBC

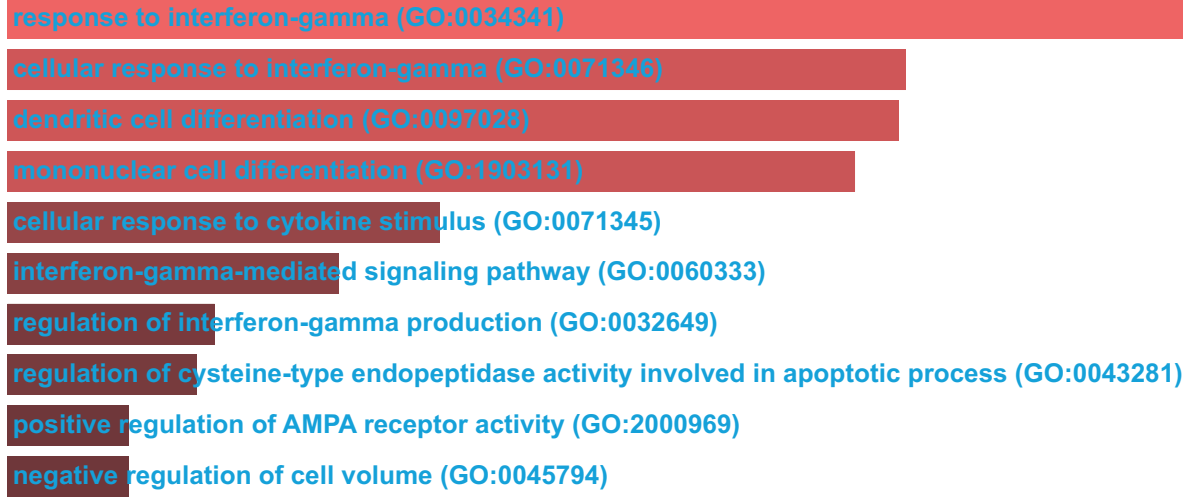

Enrichr GO Biological Process 2021 and shortlisted gene sets were also highly enriched in mutant RUNX1 AML samples (Fig. 2B and Supplementary Table 6). Interestingly, the RUNX1 mutation gene sets were also able to distinguish AML cases with the RUNX1-RUNX1T1 fusion from patients with other Runt domain mutations (Fig. 2C and Supplementary Table 7). This observation supports the notion of functional differences between these 2 types of RUNX1 mutations as recently reported by Kellaway et al. [9]. 
Fig. 2 Analysis of Awad and other RUNX1 mutant signatures in CML LBC and in AML. A Table shows GSEA analysis of 1 mutant RUNX1 CD34 + LBC sample against three wild type RUNX1 CD34 + LBC samples for the RUNX1 gene sets indicated. NES scores are denoted in red for upregulation and blue for downregulation. B GSEA heatmap shows expression of the upregulated Awad signature gene set in the left panel and TONKS_TARGETS_OF_RUNX1_RUNX1T1_FUSION_HSC_DN gene set in the right panel. 21 RUNX1 mutant (Mut) TCGA AML patient samples were compared against 130 wild type cases in red for upregulation and blue for downregulation. C GSEA heatmaps show the same gene sets with enrichments in 7 RUNX1-RUNX1T1 TCGA AML patients compared to 14 other patients with RUNX1 mutations predominantly in the Runt domain. D Bar chart shows pathway analysis of mutant RUNX1-regulated lymphoid genes aberrantly expressed in predominantly mutant RUNX1 MBC patient samples. Conserved leading edge RUNX1 mutant target genes were identified from the Awad cohort and predominantly MBC CML patients in this study (Fig. 1B). 22 RUNX1 mutation-regulated lymphoid genes were then shortlisted by overlapping with the leading edge of the wild type RUNX1 LBC versus wild type RUNX1 MBC comparison (Fig. 1C) and subjected to Enrichr [12] analysis. The top 10 most significant GO terms are inversely ranked by relative $p$-values.

Next, to understand the potential biological significance of mutant RUNX1-regulated lymphoid genes that were aberrantly expressed in mutant RUNX1 MBC samples, we performed pathway analysis on 22 mutant RUNX1 lymphoid targets identified from our analysis and found that interferon gamma signalling was highly significant (Fig. 2D). Thus, the lymphoid genes dysregulated by mutant RUNX1 in $\mathrm{MBC}$ may be involved in immune signalling and inflammation, both of which are important hallmarks of BC transformation [4].

Finally, we confirmed that the gene signatures in our RUNX1 mutant samples are enriched specifically for RUNX1 gene sets as neither RUNX2 nor RUNX3 datasets enrich significantly in RUNX1 mutant compared to wild type (Fig. 1D and Supplementary Table 2). Therefore, in addition to the Awad signature, the RUNX1 gene sets we identified can be used for more targeted studies of RUNX1 mutant signatures in CML and AML samples. These refinements may lead to better understanding of the molecular underpinnings of $\mathrm{BC} C \mathrm{CML}$ cases at risk of progressing or becoming resistant to TKI therapies, and also for mutated RUNX1 AML cases that have poor outcomes. Moreover, these patients may be amenable to new treatments specific to the RUNX1 mutational context such as through the use of BET inhibitors or proteolysis targeting chimeras (PROTACs) [10].

Kian Leong Lee $\mathbb{D D}^{1 凶}$, Tun Kiat Ko ${ }^{1,2}$, Nicole Y. L. Saw ${ }^{3}$, Asif Javed ${ }^{3,4}$, Axel M. Hillmer ${ }^{4,5}$, Charles Chuah ${ }^{1,6}$, Vaidehi Krishnan ${ }^{1}$ and S. Tiong Ong (D) ${ }^{1,6,7,8}$ -

${ }^{1}$ Cancer \& Stem Cell Biology Signature Research Programme, DukeNUS Medical School, Singapore, Singapore. ${ }^{2}$ Laboratory of Cancer Epigenome, National Cancer Centre Singapore, Singapore, Singapore. ${ }^{3}$ School of Biomedical Sciences, Li Ka Shing Faculty of Medicine, The University of Hong Kong, Hong Kong SAR, China. ${ }^{4}$ Cancer Therapeutics \& Stratified Oncology, Genome Institute of Singapore, Singapore, Singapore. ${ }^{5}$ Institute of Pathology, Faculty of Medicine and University Hospital Cologne, University of Cologne, Cologne, Germany. ${ }^{6}$ Department of Haematology, Singapore General Hospital, Singapore, Singapore. ${ }^{7}$ Department of Medical Oncology, National Cancer Centre Singapore, Singapore, Singapore.

${ }^{8}$ Department of Medicine, Duke University Medical Center, Durham,

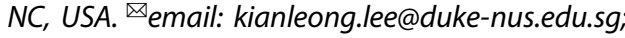
sintiong.ong@duke-nus.edu.sg

\section{REFERENCES}

1. Adnan Awad S, Dufva O, lanevski A, Ghimire B, Koski J, Maliniemi $P$, et al. RUNX1 mutations in blast-phase chronic myeloid leukemia associate with distinct phenotypes, transcriptional profiles, and drug responses. Leukemia. 2021;35:1087-99.

2. Branford S, Kim DDH, Apperley JF, Eide CA, Mustjoki S, Ong ST, et al. Laying the foundation for genomically-based risk assessment in chronic myeloid leukemia. Leukemia. 2019;33:1835-50.

3. Zhao $L$, Wang YY, Li G, Ma LY, Xiong SM, Weng XQ, et al. Functional features of RUNX1 mutants in acute transformation of chronic myeloid leukemia and their contribution to inducing murine full-blown leukemia. Blood. 2012;119:2873-82.

4. Ko TK, Javed A, Lee KL, Pathiraja TN, Liu X, Malik S, et al. An integrative model of pathway convergence in genetically heterogeneous blast crisis chronic myeloid leukemia. Blood. 2020;135:2337-53.
5. Tanaka T, Mitani K, Kurokawa M, Ogawa S, Tanaka K, Nishida J, et al. Dual functions of the AML1/Evi-1 chimeric protein in the mechanism of leukemogenesis in t(3;21) leukemias. Mol Cell Biol. 1995;15:2383-92.

6. Subramanian A, Tamayo P, Mootha VK, Mukherjee S, Ebert BL, Gillette MA, et al. Gene set enrichment analysis: a knowledge-based approach for interpreting genome-wide expression profiles. Proc Natl Acad Sci USA. 2005;102:15545-50.

7. Liu T, Ortiz JA, Taing L, Meyer CA, Lee B, Zhang $Y$, et al. Cistrome: an integrative platform for transcriptional regulation studies. Genome Biol. 2011;12: R83.

8. Cancer Genome Atlas Research N, Ley TJ, Miller C, Ding L, Raphael BJ, Mungall AJ, et al. Genomic and epigenomic landscapes of adult de novo acute myeloid leukemia. N. Engl J Med. 2013;368:2059-74.

9. Kellaway SG, Keane P, Edginton-White B, Regha K, Kennett E, Bonifer C. Different mutant RUNX1 oncoproteins program alternate haematopoietic differentiation trajectories. Life Sci Alliance. 2021;4:e202000864.

10. Mill CP, Fiskus W, DiNardo CD, Qian Y, Raina K, Rajapakshe $K$, et al. RUNX1targeted therapy for AML expressing somatic or germline mutation in RUNX1. Blood. 2019;134:59-73.

11. Tonks A, Pearn L, Musson M, Gilkes A, Mills KI, Burnett AK, et al. Transcriptional dysregulation mediated by RUNX1-RUNX1T1 in normal human progenitor cells and in acute myeloid leukaemia. Leukemia. 2007;21:2495-505.

12. Kuleshov MV, Jones MR, Rouillard AD, Fernandez NF, Duan Q, Wang Z, et al. Enrichr: a comprehensive gene set enrichment analysis web server 2016 update. Nucleic Acids Res. 2016;44:W90-97.

\section{AUTHOR CONTRIBUTIONS}

KLL and STO conceived and designed the study. KLL, VK and STO wrote and revised the manuscript. TKK performed experiments. CC provided clinical samples and information. KLL, TKK, NYLS, AJ, AMH and VK analysed data and performed statistical analyses. KLL, TKK, NYLS and VK generated figures. All authors read the manuscript and approved the submission.

\section{FUNDING}

This work was supported by grants from the National Medical Research Council Singapore (NMRC/CIRG/1468/2017 and MOH-CSASI18may-0002).

\section{COMPETING INTERESTS}

CC reports receiving honoraria from Bristol-Myers Squibb, Novartis, and Korea Otsuka Pharmaceutical and research funding from Bristol-Myers Squibb. STO has received royalties from AUM Biosciences. The remaining authors declare no competing interests.

\section{ADDITIONAL INFORMATION}

Supplementary information The online version contains supplementary material available at https://doi.org/10.1038/s41375-022-01508-1.

Correspondence and requests for materials should be addressed to Kian Leong Lee or S. Tiong Ong.

Reprints and permission information is available at http://www.nature.com/ reprints

Publisher's note Springer Nature remains neutral with regard to jurisdictional claims in published maps and institutional affiliations. 
(c) Open Access This article is licensed under a Creative Commons

Attribution 4.0 International License, which permits use, sharing, adaptation, distribution and reproduction in any medium or format, as long as you give appropriate credit to the original author(s) and the source, provide a link to the Creative Commons license, and indicate if changes were made. The images or other third party material in this article are included in the article's Creative Commons license, unless indicated otherwise in a credit line to the material. If material is not included in the article's Creative Commons license and your intended use is not permitted by statutory regulation or exceeds the permitted use, you will need to obtain permission directly from the copyright holder. To view a copy of this license, visit http://creativecommons. org/licenses/by/4.0/.

(c) The Author(s) 2022 